\title{
Memory or amnesia: the dilemma of stem cell therapy in muscular dystrophies
}

\author{
Marco Sandri \\ Venetian Institute of Molecular Medicine (VIMM), Padova, Italy. Department of Biomedical Sciences, University of Padova, Padova, Italy. Consiglio Nazionale delle Ricerche (CNR), Institute of Neuroscience, \\ Padova, Italy. Department of Medicine, McGill University, Montreal, Quebec, Canada.
}

\begin{abstract}
Muscular dystrophies are monogenetic diseases that are often characterized by the degeneration of both cardiac and skeletal muscle. Gene therapy to correct the mutated gene has shown promise in both animal models and clinical trials; however, current gene delivery strategies are limited to the introduction of the corrected gene into only one tissue. Strategies to target multiple striated muscle types would provide a much-needed improvement for the treatment of muscular dystrophies. In this issue of the $J C l$, Quattrocelli and colleagues demonstrate that induced pluripotent stem cells (iPSCs) with a myogenic propensity are able to engraft into both cardiac and skeletal muscles. The authors also identified a novel pool of mesodermal iPSC-derived progenitors (MiPs). Moreover, the authors show that these MiPs are amenable to gene correction and can restore function in murine dystrophic models. Together, the results of this study provide an important advance in improving gene delivery to treat patients with muscular dystrophy.
\end{abstract}

\section{Harnessing the potential of stem cells for regenerative therapy}

The presence of pluripotent or multipotent stem cells in tissues has long inspired scientists to explore these cells for their potential application in medicine. Several therapeutic uses for these cells have been proposed and are currently under investigation. For example, stem cells could be used to generate organs in vitro for transplantation, autologous cells could possibly regenerate tissue in vivo, or these cells could be harnessed to correct inherited diseases. Although the ability to build artificial and functional organs, like liver or heart, is far from being achieved, the therapeutic application of stem cells in regenerative medicine and hereditary disorders is already in advanced stages $(1,2)$.

Muscular dystrophies are inherited genetic diseases that are particularly chal- lenging from a therapeutic point of view. As striated muscles are formed by syncytial postmitotic cells that have limited or absent regenerative potential, muscle is not readily reconstructed. Moreover, the human body contains more than 600 striated muscles that are widely distributed, and these muscles control vital functions such as respiration, chewing and swallowing for food intake, and blood circulation. Muscles also regulate sugar and lipid metabolism, support the vertebrae, avoiding collapse or curving of the spinal column, and allow locomotion. All these functions are compromised in inherited muscular dystrophies, because both cardiac and skeletal muscles are often simultaneously affected. So far, the majority of preclinical and clinical therapeutic trials aimed at harnessing the regenerative properties of stem cells have focused on restoring just one tissue, heart or skeletal muscle (3). Therefore, a

Related Article: p. 4463

Conflict of interest: The author has declared that no conflict of interest exists.

Reference information: / Clin Invest. 2015;125(12):4331-4333. doi:10.1172/JCI85002.

strategy that would allow for the combined regeneration of both striated muscle types would constitute a major advancement in the treatment of muscular dystrophies.

A critical step for any potential gene therapy for muscular dystrophies is the delivery of the corrected gene to a tissue that accounts for $40 \%$ to $50 \%$ of whole body mass. In this regard, adult stem cells have become an attractive vector for gene delivery and a valid option for viral vectors. Such a cell-based therapeutic strategy requires several steps that involve the purification of adult stem cells from the patient, correction of the genetic defect, expansion of the gene-corrected cells, and reinjection of the desired cell population into the patient. Following reinjection, fusion of stem cells into myofibers allows delivery of the gene and correction of the genetic abnormality. Even though this sequence of events appears relatively straightforward, several issues and challenges must be overcome that are primarily related to the enormous number of adult stem cells that are required for this approach. Adult stem cells are difficult to expand and only reach the numbers required for cell therapy in humans if clonal expansion is performed (4), which increases the risk of tumor formation once the cells are transplanted into the patient. The unlimited self-renewal capacity of induced pluripotent stem cells (iPSCs) (5) overcomes the obstacle of sufficient numbers but does not solve all the technical issues involved in cell therapy. In fact, epigenetic modifications are acquired during development and become stable and inherited. These changes are termed "epigenetic memory" and are used to characterize the differentiation state of the cell (6). The reprogramming of somatic cells back to their pluripotent state requires a complete erasure of the existing somatic epigenetic memory in order to establish a new signature. Recent evidence indicates that epigenetic biases are maintained during reprogramming, as tissue-specific histone modifications are often observed in 


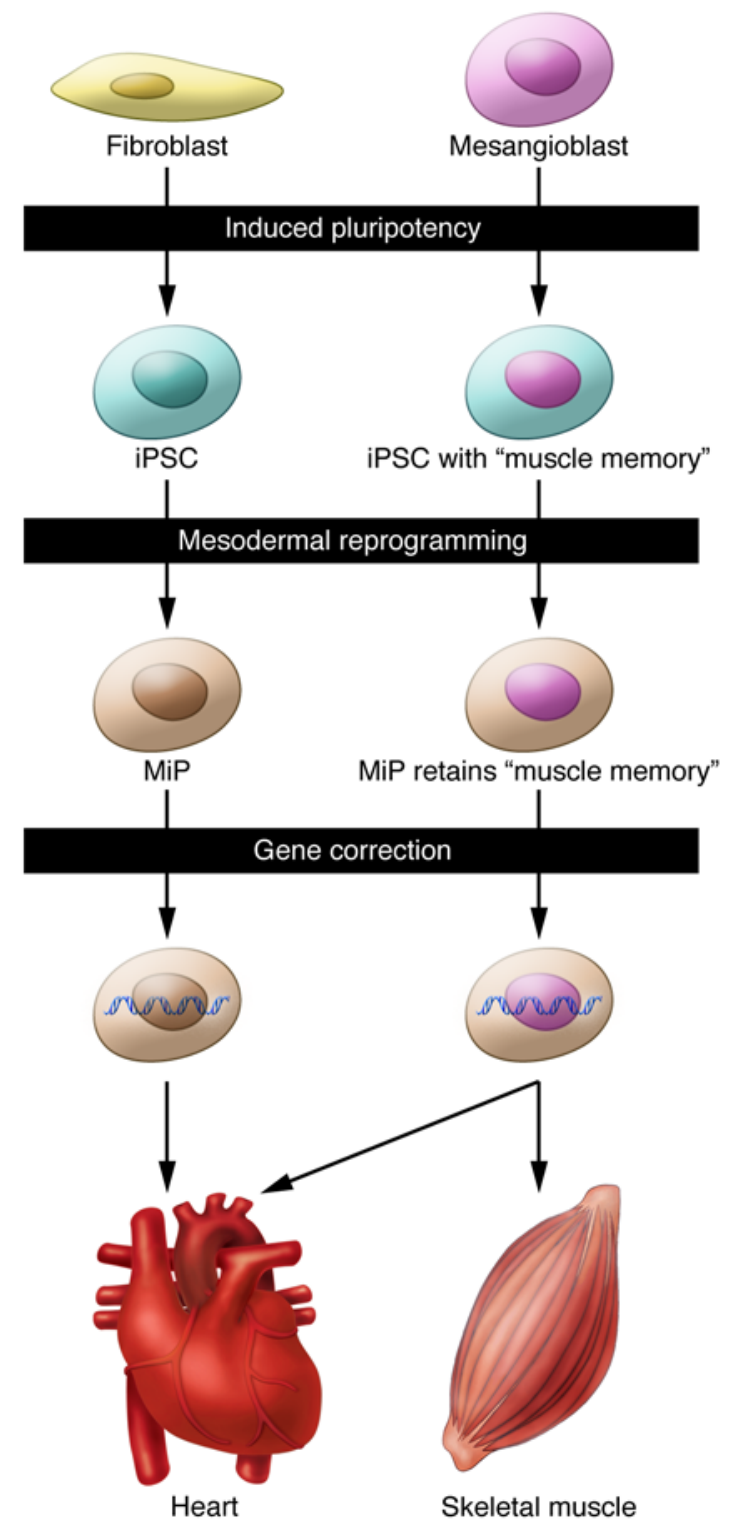

Figure 1. MiPs carrying the corrected DNA have the potential to repair damaged striatal muscle. The decision regarding where MiPs migrate and engraft is greatly affected by the cell's memory. Independent of their origin and memory, fibroblast- and mesangioblast-derived MiPs both preferentially target the heart, while only the cells that have a certain memory of the muscle environment are able to reach and engraft into skeletal muscles.

iPSCs (6); therefore, epigenetic memory is a critical barrier during the reprogramming process. For this reason, the cell type used for iPSC generation and DNA correction may become critical for a successful cell therapy that targets both heart and skeletal muscles.

\section{Tissue-specific biases improve engraftment of pluripotent stem cells into skeletal muscle} While tissue-specific biases can be decreased by continuous iPSC passaging (7), it is not clear whether complete memory loss must be achieved to obtain better muscle engraftment. In fact, it has recently been shown that iPSCs with some remnants of muscle origin have improved myogenic differentiation capacity (8); however, it has not been fully explored whether biased cells are an obstacle or an advantage in iPSC-mediated therapy for muscular dystrophies. In this issue, Quattrocelli and colleagues (9) address the relevance of the tissue of provenance/ origin of iPSCs for muscle regeneration. Specifically, Quattrocelli et al. generated iPSCs from fibroblasts and mesan- gioblasts, which are pericyte-like cells with an intrinsic myogenic propensity, and evaluated the effects of the iPSC subtypes on both cardiac and skeletal muscles. The results of this study clearly show that muscle memory is essential for an efficient iPSC engraftment into skeletal muscle but is dispensable for engraftment into the heart. Using murine models, Quattrocelli and colleagues found that both fibroblastand mesangioblast-derived iPSCs efficiently incorporated into damaged cardiac muscle, while mesangioblast iPSCs preferentially engrafted into injured skeletal muscle. The authors also identified a novel pool of mesodermal iPSC-derived progenitors (MiPs) that they were able to isolate from developing muscles of murine, canine, and human origin. Importantly, the intrinsic myogenic propensity of these cells did not affect their pluripotency, as both iPSCs and MiPs efficiently differentiated and incorporated into several tissues, including liver, skin, adipose tissue, and heart. Moreover, the inherent striatal muscle bias of MiPs was conserved between species, and both canine and human MiPs better differentiated into muscles due to their intrinsic myogenic propensity. Therefore, an efficient therapy to target striated muscles must take into consideration the myogenic memory of the iPSCs or MiPs that are used.

\section{Remaining questions and future considerations}

Although these findings are a major advance toward successful cell therapy for muscular dystrophies, several points still need to be addressed in future studies. It is well known that satellite cells, stem cells of muscle tissue, are not good candidates for iPSC reprogramming; however, it is now clear that a certain reminiscence of the muscle environment is crucial for an efficient engraftment into skeletal muscles (Figure 1). Independent of their origin, MiPs display some markers of myogenesis, such as muscle-specific miRs, but still have a low myogenic propensity when derived from reprogrammed fibroblasts. Indeed, Quattrocelli and colleagues found that the myogenic bias of MiPs is consequent to the maintenance of specific DNA methylation signatures and histone marks on some muscle-specific genes. However, only a relatively small number of genes 
were monitored for epigenetic changes, and, therefore, a better understanding of the epigenetic alterations within the entire genome that allow for efficient muscle engraftment would be an important step in optimizing cell therapy for muscular dystrophies.

A second important point raised by the study by Quattrocelli and colleagues (9) is the impact that iPSC-based procedures and DNA correction have on the genome. In fact, the substantial chromatin changes that are required to establish pluripotency may also promote DNA fragility. Indeed, a recent clinical trial that used iPSCs to generate specialized retinal cells for the treatment of age-related macular degeneration was paused because unintended mutations were found in the reprogrammed cells. The risk of introducing de novo mutations is even greater during the DNA-editing process because of off-target effects associated with both the TALEN (10) and CRISPR/Cas 9 systems $(11,12)$. These technologies have been used in iPSCs to restore the proper coding sequence via exon skipping, frame shifting, or exon knockin and have resulted in minimal, but not absent, off-target effects (13). Quattrocelli and colleagues used the TALEN system in MiPs that they derived from a canine model of muscular dystrophy to correct the mutated dystrophin gene and restore functional protein. Moreover, the introduction of corrected canine-derived MiPs functionally restored muscle function in dystrophic mice, confirming that MiPs are suitable for DNA editing and gene therapy. However, this study did not evaluate whether or not additional mutations were introduced into the genome of the corrected cells. Certainly, iPSCs and/or MiPs are the future of cell-based regenerative therapy; however, caution must be exercised as TALEN, CRISPR/Cas9, and the generation of pluripotency itself can promote de novo mutations. Therefore, it is mandatory to perform whole-genome sequencing after DNA editing in iPSCs or MiPs that have potential to be used in patients.

Finally, despite the successful engraftment of murine and reprogrammed canine MiPs into multiple striated muscle types of sarcoglycan-deficient mice, it should be noted that the dystrophin deficiency and phenotypes in humans are more severe than those found in mice. Indeed, patients with Duchenne muscular dystrophy rapidly lose muscle function and become wheelchair dependent at a young age. It is unlikely that cell therapy will restore functionality to patients who are severely affected and have relatively few muscle cells. As successful cell therapy requires the presence of myofibers, treatment of patients with MiPs or iPSCs must start as early as possible in order to allow the fusion of pluripotent cells with the remaining myofibers to correct the genetic defect. The presence of fibrotic and adipose tissues in severely affected individuals also constitutes a major barrier for cell diffusion and fusion and can therefore prevent efficient muscle regeneration. Strategies that induce expression of the fusion protein myomaker in MiPs and iPSCs (14) might provide added benefit and affect the efficacy of cell therapy. In conclusion, the study by Quattrocelli et al. and other recent studies introduce substantial advances to the cell therapy field and offer new hope for a cure for patients affected by muscular dystrophies.

\section{Acknowledgments}

The author's research is supported by the European Research Council (282310-MyoPHAGY), the Italian Ministry of Education (MiUR) (PRIN 2010/2011), and the Foundation Leducq.
Address correspondence to: Marco Sandri, Address: via Orus 2, 35129 Padova, Italy. Phone: 0039.049.7923258; E-mail: marco. sandri@unipd.it.

1. Kimbrel EA, Lanza R. Current status of pluripotent stem cells: moving the first therapies to the clinic. Nat Rev Drug Discov. 2015;14(10):681-692.

2. Fisher SA, Doree C, Mathur A, Martin-Rendon E. Meta-analysis of cell therapy trials for patients with heart failure. Circ Res. 2015;116(8):1361-1377.

3. Mercuri E, Muntoni F. Muscular dystrophies. Lancet. 2013;381(9869):845-860.

4. Ousterout DG, et al. Reading frame correction by targeted genome editing restores dystrophin expression in cells from Duchenne muscular dystrophy patients. Mol Ther. 2013;21(9):1718-1726.

5. Takahashi K, et al. Induction of pluripotent stem cells from adult human fibroblasts by defined factors. Cell. 2007;131(5):861-872.

6. Nashun B, Hill PW, Hajkova P. Reprogramming of cell fate: epigenetic memory and the erasure of memories past. EMBO J. 2015;34(10):1296-1308.

7. Polo JM, et al. Cell type of origin influences the molecular and functional properties of mouse induced pluripotent stem cells. Nat Biotechnol. 2010;28(8):848-855.

8. Quattrocelli M, et al. Intrinsic cell memory reinforces myogenic commitment of pericytederived iPSCs. J Pathol. 2011;223(5):593-603.

9. Quattrocelli M, et al. Mesodermal iPSCderived progenitor cells functionally regenerate cardiac and skeletal muscle. JClin Invest. 2015;125(12):4463-4482.

10. Hockemeyer D, et al. Genetic engineering of human pluripotent cells using TALE nucleases. Nat Biotechnol. 2011;29(8):731-734.

11. Cong L, et al. Multiplex genome engineering using CRISPR/Cas systems. Science. 2013;339(6121):819-823.

12. Mali $P$, et al. RNA-guided human genome engineering via Cas9. Science. 2013;339(6121):823-826.

13. Li HL, et al. Precise correction of the dystrophin gene in duchenne muscular dystrophy patient induced pluripotent stem cells by TALEN and CRISPR-Cas9. Stem Cell Reports. 2015;4(1):143-154.

14. Millay DP, et al. Myomaker is a membrane activator of myoblast fusion and muscle formation. Nature. 2013;499(7458):301-305. 